\title{
The formation of nanograin structures and accelerated room-temperature theta precipitation in a severely deformed $\mathrm{Al}-4$ wt.\% $\mathrm{Cu}$ alloy
}

\author{
Y. Huang, J.D. Robson, P.B. Prangnell* \\ Manchester Materials Science Centre, The University of Manchester, Grosvenor Street, Manchester M17HS, UK
}

Received 4 September 2009; received in revised form 6 November 2009; accepted 6 November 2009

Available online 16 December 2009

\begin{abstract}
The grain size achievable and long-term stability of a severely deformed aluminium copper alloy have been investigated when copper is used in solution to inhibit recovery. It is shown that copper is more effective than magnesium in inhibiting dynamic recovery. A grain width of only $\sim 70 \mathrm{~nm}$ was obtained in an $\mathrm{Al}-4 \mathrm{wt} . \% \mathrm{Cu}$ alloy, after processing by equal-channel angular extrusion to a strain of $\varepsilon_{\mathrm{eff}}=10$, resulting in a lamellar nanograin structure. However, post-processing, the severely deformed solid solution was found to be unstable at room temperature and copious precipitation of $\theta$ occurred at grain boundaries within the deformed state, leading to recovery of the deformation structure and a loss of strength. The solute level fell to equilibrium within $\sim 9$ months. The precipitation kinetics were shown to occur at many orders of magnitude higher than can be predicted by classical nucleation and growth theory. The reasons for this discrepancy are discussed.
\end{abstract}

(C) 2009 Acta Materialia Inc. Published by Elsevier Ltd. All rights reserved.

Keywords: Severe plastic deformation (SPD); Nanocrystalline; Accelerated ageing; Theta phase; Nucleation

\section{Introduction}

There is currently considerable interest in the production of ultrafine-grained (UFG) metals with nanograin structures by severe plastic deformation (SPD) (e.g. Refs. [1-21]). This interest stems not only from the practical viewpoint of economically obtaining materials with enhanced mechanical performance, and/or finer scale microstructural homogeneity, but also from the desire to extend our knowledge of the evolution of deformation structures to ultra-high strains and their subsequent stability (e.g. Refs. [1-15]). Although various methods can be used to produce materials with nanoscale grain sizes, techniques like equal-channel angular extrusion (ECAE) [16,17] have the advantage of a "top down" approach, involving grain refinement from a bulk billet by ultra-high strain deformation [1-7]. SPD processing is

\footnotetext{
${ }^{*}$ Corresponding author. Tel.: +44 161306 3610; fax: +44 1613063586 . E-mail address: philip.prangnell@manchester.ac.uk (P.B. Prangnell).
}

thus free from the problems of residual porosity and contamination that are frequently encountered when materials are produced by "bottom up" techniques requiring, for example, the consolidation of atomized powders.

In SPD processing it is becoming increasingly recognized that a steady state grain size is eventually reached at ultrahigh strains that limits the level of grain refinement that can be achieved [3-6]. This limit is controlled by dynamic recovery operating at surprisingly low temperatures and a far higher rate than seen statically $[3,6,18]$. It is thus difficult to obtain a nanograin structure in aluminium alloys on processing under ambient conditions owing to the relatively high homologous temperature that results from their low melting point. Methods available for decreasing the limiting grain size include cryogenic deformation [3,12], the introduction of second-phase particles to pin boundary migration $[8,19]$, or the use of solute to inhibit recovery [20,21]. Of these options, in aluminium alloys, the use of solute is arguably the most effective approach, as cryogenically deformed 
nanograined aluminium alloys are unstable even at room temperature [3] and it is difficult to achieve the required particle densities with conventional ingot metallurgy $[8,19]$. In aluminium metallurgy, magnesium is the alloying element most widely used to provide solid solution strengthening and increase work hardening by reducing the rate of recovery. Alloying with magnesium has also been shown to greatly reduce the grain size in SPD-processed aluminium [11,15,21]. Grain widths of $\sim 100 \mathrm{~nm}$ have been measured in an Al-3 wt. $\% \mathrm{Mg}$ alloy (3.3 at.\%), processed to a strain of ten by ECAE compared to $\sim 500-600 \mathrm{~nm}$ in a dilute Al-0.1 wt.\% Mg alloy [11]. However, similar to cryogenic deformation [3], when a directional SPD processing route is used, the reduced rate of dynamic boundary migration results in a far more elongated grain structure than seen in more dilute aluminium alloys $[6,11]$. For example, in the results cited above, the grain aspect ratio rose from 2.3 to 7 on increasing the $\mathrm{Mg}$ level to $3 \mathrm{wt} . \%$ [11].

Magnesium has long been known to inhibit dynamic recovery in aluminium alloys $[22,23]$ and this has often been associated with a reduction in the stacking fault energy (SFE) restricting cross slip. However, the main effect, on inhibiting cell structure formation during recovery, is probably from the drag of solute atmospheres and the thermal activation of solute atoms away from climbing jogs or screw dislocation segments, as the dissociation of partials is small in $\mathrm{Al}-\mathrm{Mg}$ solid solutions [24,25]. Relative to magnesium, the influence of copper in aluminium solid solutions on recovery has received less attention. While copper increases the SFE [26], when compared at the same atomic concentration, it has been found to have a greater influence on both solid solution strengthening and reducing the rate of recovery, at least after deformation to conventional strains [24]. Although data in the correct concentration ranges is scarce, $\mathrm{Cu}$ and $\mathrm{Mg}$ appear to have similar effects on retarding grain boundary mobility $[27,28]$. Furthermore, in one study grain sizes as fine as $\sim 100 \mathrm{~nm}$ have been reported in an $\mathrm{Al}-4 \mathrm{wt} . \%$ $\mathrm{Cu}$ alloy (1.7 at.\%) deformed to an effective strain of $\sim 9$ by ECAE [29], which is lower than that noted above for higher atomic concentrations of magnesium.

An important limitation on the use of solute to inhibit dynamic recovery and achieve nanograin structures in SPD is the stability of supersaturated solid solutions during and after severe deformation. Straumal et al. [30,31] have investigated the stability of $\mathrm{Al}-\mathrm{Mg}$ and $\mathrm{Al}-\mathrm{Zn}$ supersaturated solid solutions during extremely high strain SPD by highpressure torsion $(\gamma \sim 300)$. They found that the $\mathrm{Al}-\mathrm{Zn}$ solid solution dynamically decomposed, approaching equilibrium, whereas with $\mathrm{Al}-\mathrm{Mg}$ only partial decomposition occurred. Straumal et al. explained this difference in terms of the higher diffusivity of zinc in aluminium, but found that the overall rate was still around $10^{8}$ times faster than could be accounted for by lattice diffusion. This enhanced rate of decomposition was attributed to grain boundary diffusion, accelerated by the very high vacancy flux produced under the dynamic SPD conditions, and the sweeping up of solute into boundaries by their deformation-induced movement. In this context it is interesting to note that copper has a higher activation energy for diffusion than either magnesium or zinc [32].

The high-angle boundary area for a $100 \mathrm{~nm}$ grain size will be of the order of 100 times that for a more normal $10 \mu \mathrm{m}$ grain size and the vacancy concentration has been measured to be as high as $10^{-4}$ immediately after SPD [33]. As a result, even when dynamic decomposition of supersaturated solid solutions does not occur, several studies have shown that the SPD accelerates precipitation on artificial ageing and can also change the precipitation sequence $[29,34]$. For example, it has been found that in an $\mathrm{Al}-1.7$ at. $\% \mathrm{Cu}$ alloy processed by ECAE to a strain of 8 , ageing at $100{ }^{\circ} \mathrm{C}$ for $24 \mathrm{~h}$ results in the equilibrium $\mathrm{Al}_{2} \mathrm{Cu}(\theta)$ phase precipitating on grain boundaries directly from solid solution, bypassing the normally seen metastable transition sequence of GP zones, $\theta^{\prime \prime}$ and $\theta^{\prime}$ [29].

As part of an ongoing project exploring the fundamental principles of producing nanocrystalline aluminium alloys by SPD, the initial objective of this study was to investigate the deformation structure evolution of $\mathrm{Al}-\mathrm{Cu}$ solid solutions during room-temperature severe deformation, with a view to exploring whether a nanograin structure could be achieved that was finer than that typically seen in $\mathrm{Al}-\mathrm{Mg}$ alloys. During the course of this work, it was accidentally discovered that the equilibrium $\mathrm{Al}_{2} \mathrm{Cu}$ theta $(\theta)$ phase precipitated copiously on grain boundaries, within the deformation structure, on long-term natural ageing at room temperature after SPD processing. To the best of the authors' knowledge, there have been no previous reports of the $\theta$ phase precipitating during natural ageing in the same, or similar alloys, even when the solid solution is extended to much higher supersaturations [38], although GP zones may readily form [35]. The $\theta$ phase nucleated and grew at a surprisingly fast rate, given the low diffusivity of copper in aluminium, and growth continued for far longer than might be expected from the time for the excess vacancy content to decrease from its artificially high postprocessing level. The present paper will thus focus on quantifying the precipitation kinetics during the room-temperature ageing of a SPD-processed $\mathrm{Al}-4 \mathrm{wt} . \% \mathrm{Cu}$, with a view to explaining this unusual accelerated ageing phenomenon.

\section{Experimental details}

A high-purity $\mathrm{Al}-4$ wt. $\% \mathrm{Cu}$ alloy $(1.7$ at. $\% \mathrm{Cu})$ was made from $99.999 \%$ pure aluminium (supplied by Alcan International) and electrical-grade copper. The alloy was cast in a $22 \mathrm{~mm}$ thick copper book-shaped mould under an argon atmosphere and homogenized at $550^{\circ} \mathrm{C}$ for $24 \mathrm{~h}$. The ingots were machined into $100 \mathrm{~mm}$ long bars with a square cross-section of $15 \times 15 \mathrm{~mm}^{2}$. The bars were subsequently solution-treated by heating to $500^{\circ} \mathrm{C}$ for $2 \mathrm{~h}$, followed by $4 \mathrm{~h}$ at $550{ }^{\circ} \mathrm{C}$, before quenching in water. The supersaturated bars were subsequently processed by ECAE immediately after solution treatment (within $20 \mathrm{~min}$ ). 
ECAE was carried out at room temperature with a ram speed of $50 \mathrm{~mm} \mathrm{~min}^{-1}$, through a $120^{\circ}$ die, using colloidal graphite as lubricant. Up to 15 passes were applied to obtain a range of strain levels, using route $\mathrm{A}$ [16]; i.e. the specimen orientation was kept constant throughout the multi-pass processing. The effective strain $\left(\varepsilon_{\mathrm{eff}}\right)$ per ECAE cycle was approximately 0.7 [17]. The deformed specimens were then naturally aged at room temperature for up to a year while their hardness and microstructures were monitored.

The deformation structures of the ECAE-processed billets and the second-phase particles, precipitated during natural ageing at room temperature, were characterized by backscatter electron (BSE) imaging and electron backscattered diffraction (EBSD) in a high-resolution FEI Sirion FEGSEM, fitted with an HKL-EBSD system. This analysis was complemented at higher resolutions by transmission electron microscopy in a Philips CM200 TEM and an FEI Tecnai F30 FEGTEM in STEM mode using a HAADF detector. X-ray diffraction was carried out to con- firm the crystal structure of the precipitates. Hardness was measured on an Instron Tukon2100 Vickers micro-hardness tester, with the application of a $200 \mathrm{~g}$ load for $10 \mathrm{~s}$. Each measurement was obtained by averaging over six individual indents. All the microstructural data presented are from near the centre of the longitudinal transverse plane (TD plane) of the ECAE billets, defined by the normal direction (ND) and the extrusion direction (ED) in reference to the ECAE deformation geometry [3]. Specimens for SEM-EBSD analysis were mechanically polished to $0.5 \mu \mathrm{m}$ before electro-polishing in a solution of $30 \%$ nitric acid in methanol. TEM thin foils were prepared by jet polishing using the same solution at $-30^{\circ} \mathrm{C}$.

\section{Results}

\subsection{Deformation structures}

Backscatter electron images in Fig. 1 show the deformation structure of the supersaturated $\mathrm{Al}-4 \mathrm{wt} . \% \mathrm{Cu}$ alloy
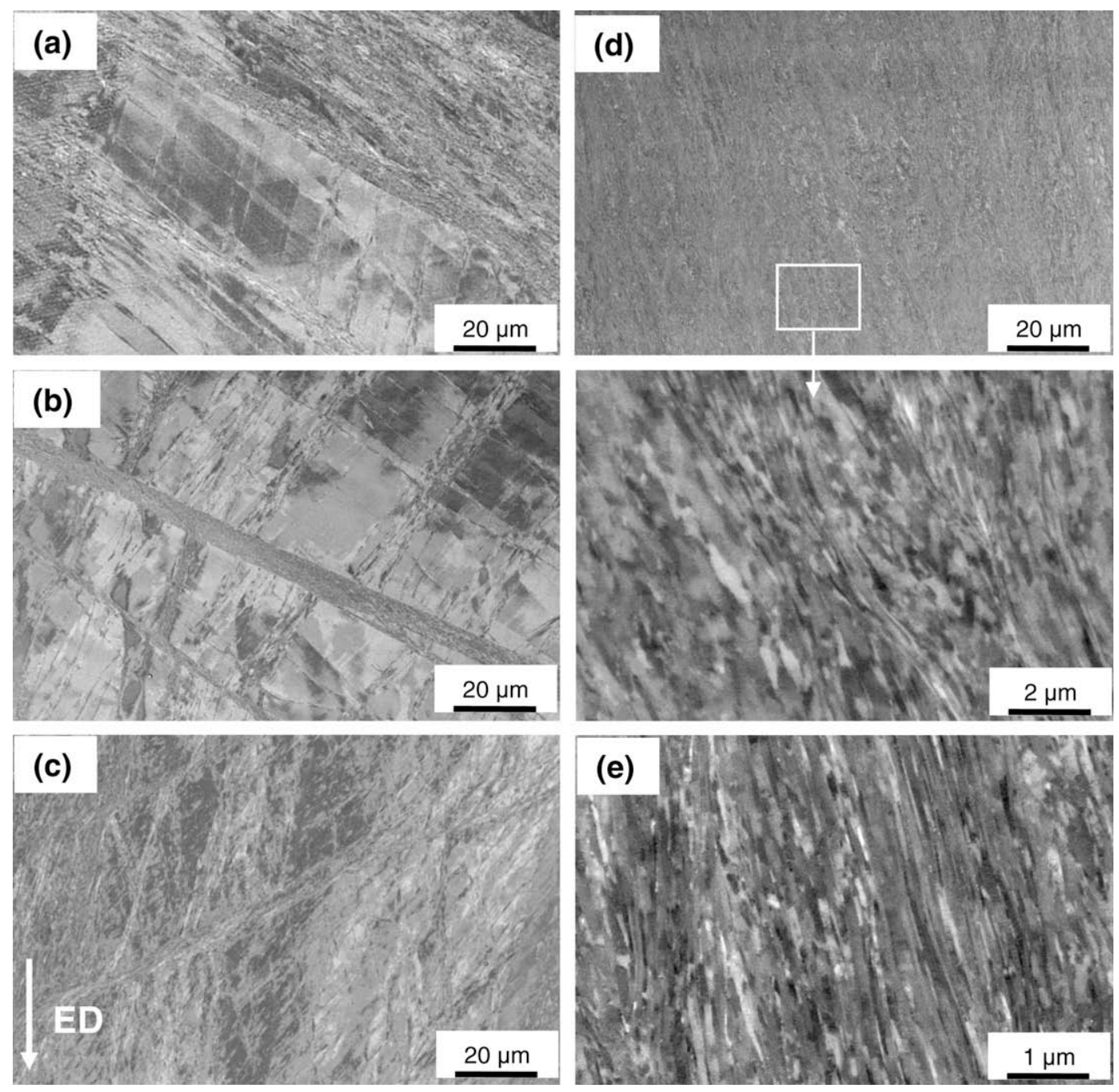

Fig. 1. Electron backscatter images of the ECAE-processed $\mathrm{Al}-4 \mathrm{Cu}$ samples, showing the deformation structures immediately after ECAE processing to: (a) one pass, (b) two passes, (c) four passes, (d) seven passes (high and low magnification), and (e) 15 passes. Note the change in magnifications with strain. 
Table 1

Average crystallite sizes measured directly after ECAE processing from backscatter images, with the approximate HAGB area fraction and mean misorientations obtained from EBSD maps following room-temperature recovery. The data in italics at low strains are only from within shear bands. Linear intercept HAGB $\left(\geqslant 15^{\circ}\right)$ spacings, determined from orientation maps of the 15 pass ECAE sample, are also included.

\begin{tabular}{|c|c|c|c|c|c|}
\hline \multirow[t]{2}{*}{$\begin{array}{l}\text { ECAE } \\
\text { pass }\end{array}$} & \multirow[t]{2}{*}{$\begin{array}{l}\text { Effective } \\
\text { strain }\end{array}$} & \multicolumn{2}{|c|}{$\begin{array}{l}\text { Crystallite size } \\
(\mathrm{nm})\end{array}$} & \multirow{2}{*}{$\begin{array}{l}\text { Approx. } \\
\text { HAGB } \\
(\%)\end{array}$} & \multirow{2}{*}{$\begin{array}{l}\text { Mean HAGB } \\
\text { misorientation } \\
\text { (degrees) }\end{array}$} \\
\hline & & $\begin{array}{l}\text { Width, } \\
\lambda_{N D}\end{array}$ & $\begin{array}{l}\text { Length, } \\
\lambda_{E D}\end{array}$ & & \\
\hline 1 & 0.67 & 186 & 235 & & \\
\hline 2 & 1.33 & 173 & 281 & 7.7 & 19 \\
\hline 4 & 2.67 & 165 & 303 & 25.6 & 24 \\
\hline 5 & 3.4 & 133 & 343 & 33.5 & 33 \\
\hline 7 & 4.67 & 127 & 408 & 57.4 & 39 \\
\hline 13 & 8.71 & 72 & 520 & 74.6 & 42 \\
\hline \multirow[t]{3}{*}{15} & 10.05 & 65 & 570 & 78.3 & 43 \\
\hline & & \multicolumn{2}{|c|}{$\begin{array}{l}\text { HAGB spacing } \\
(\mathrm{nm})\end{array}$} & & \\
\hline & & $\begin{array}{l}\text { Width, } \\
\lambda_{N D}\end{array}$ & $\begin{array}{l}\text { Length, } \\
\lambda_{E D}\end{array}$ & & \\
\hline 15 & 10.05 & 80 & 1000 & 78.3 & 43 \\
\hline
\end{tabular}

immediately after processing for increasing numbers of ECAE passes. Due to the fine nature of the deformation structure, and high internal strains, it proved extremely difficult to obtain EBSD maps with a sufficient level of indexing to provide reliable data directly after processing. Indexing was improved sufficiently to give reasonable information after leaving the samples to recover for 10 months' at room temperature, following processing. However, the level of indexing of the EBSD maps was still relatively low in shear bands and highly deformed areas. At low strains $\left(\varepsilon_{\text {eff }}<3\right)$ a cellular substructure could only be resolved by BSE imaging within shear bands and not in the matrix. The grain/subgrain, or crystallite, sizes of the samples were thus measured immediately after processing (within $4 \mathrm{~h}$ ) from BSE images, where clear cells could be resolved, without discriminating between high- and low-angle boundaries using the mean linear intercept method. The fraction and mean misorientation angle of high-angle grain boundaries (HAGBs) were determined from EBSD orientation maps, after natural ageing, but would not be expected to be greatly affected by the limited recovery that occurred (see Section 3.4). These microstructural parameters are given in Table 1, as a function of the number of ECAE passes. At high strains $\left(\varepsilon_{\mathrm{eff}}=10\right)$ it was possible to measure statistically reliable high-angle boundary spacings $\left(\mathrm{HAGB} \geqslant 15^{\circ}\right.$ ) from the EBSD orientation maps after 10 months' room-temperature recovery (e.g. Fig. 2c) and this data is also given in Table 1. The measurement of crystallite sizes from the BSE images was crosschecked using TEM and the results from both techniques were in good agreement (see for example Fig. 10).

In the early stages of processing, deformation was highly heterogeneous and shear bands were the main characteristic feature of the deformation structure. As shown in Fig. 1a, in the first pass of ECAE, the majority of strain was carried within shear bands aligned with the die shear plane $\left(60^{\circ}\right.$ to $\left.\mathrm{ED}\right)$. Shear banding was also dominant in the second pass and it can be seen in Fig. $1 b$ that more intense shear bands have formed after two passes, which cut through the weaker shear band structure formed in the first pass at an angle of $60^{\circ}$. The primary shear bands generated in each pass (parallel to the shear plane) tended to form in conjunction with a complementary set of slip lines, or a set of relatively weaker shear bands, intersecting at an angle of $60-70^{\circ}$ (Fig. 1b).

On processing to higher strains, the shear band structures formed in previous ECAE passes were compressed and rotated towards the extrusion direction and deformation became more uniform as severe strain localization reduced (Fig. 1). After seven passes, individual band structures were no longer distinguishable from each other and a fine lamellar structure formed, as shown in Fig. 1d. With higher strains, the grain boundary spacing was further compressed and eventually a more uniform, finer, and highly elongated lamellar grain structure developed by a strain of 10 (Fig. 1e).

EBSD measurements showed that shear banding generated both new low- and high-angle grain boundaries. Fig. 2a depicts an EBSD orientation map obtained from a sample processed by two ECAE passes, showing new high-angle grain boundaries mainly associated with shear bands aligned in the ECAE die's shear plane. There are also HAGBs within shear bands formed in the first pass. More new HAGBs were generated within shear bands after four passes (Fig. 2b) and after 15 passes the deformation structure was dominated by HAGBs, although at higher strains shear banding was less significant and the geometrical increase in HAGB area required by the strain tensor became more important (Fig. 2c).

The data summarized in Table 1 shows that that after deformation to an effective strain of ten the average crystallite size was only $65 \mathrm{~nm}$ wide by $\sim 500 \mathrm{~nm}$ long, although the length was more difficult to determine reliably as there were some very low misorientation transverse boundaries present within the lamellar structures (see TEM images in Fig. 6). When it was possible to obtain EBSD maps after 10 months' room-temperature recovery, Fig. $2 b$ shows that the HAGB structure still appeared highly lamellar in nature, and contained very high aspect ratio grain fragments and few transverse HAGBs. In the EBSD maps the average lamellar HAGB spacing was measured to be $\lambda_{\mathrm{ND}}=80 \mathrm{~nm}$, which is in close agreement with the other data and confirms that the lamellar boundaries were predominantly high-angle in character. However, it can be seen that some of the lamellar grains extend over several microns and the average length, or HAGB spacing in ED, was estimated to be $\lambda_{\mathrm{ED}} \sim 1 \mu \mathrm{m}$, giving an estimated grain aspect ratio of $\sim 13-15$ after processing.

\subsection{Micro-hardness response during natural ageing}

In Fig. 3 the natural ageing response of samples processed to five and 15 ECAE passes are compared to a 

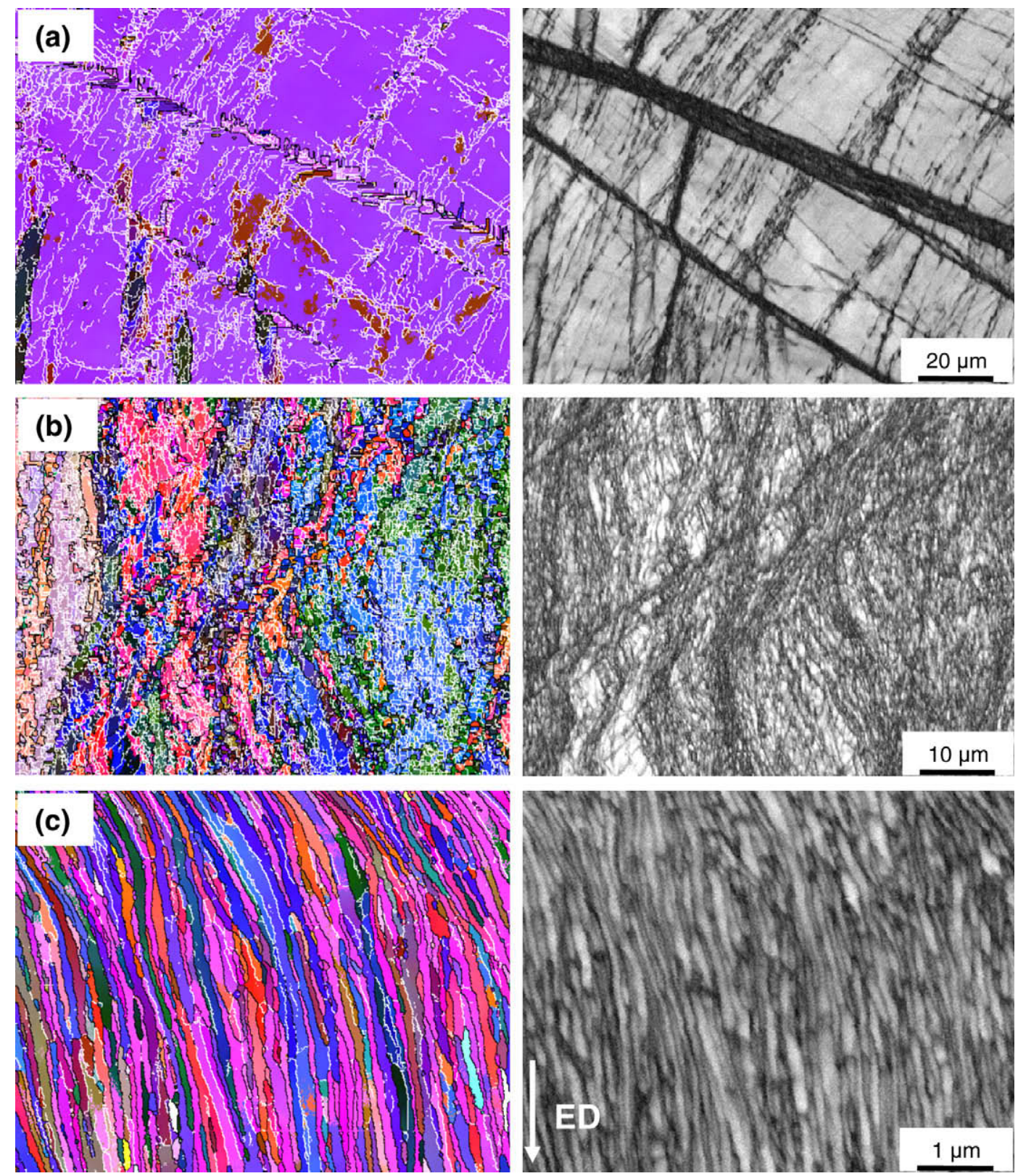

Fig. 2. EBSD orientation and pattern quality maps obtained from samples processed by ECAE to: (a) two passes (b) four passes and (c) 15 passes (after 10 months' natural ageing). In the orientation maps HAGBs $\left(\geqslant 15^{\circ}\right)$ are represented by black lines and low-angle boundaries by white lines.

solution-treated undeformed control, on natural ageing at $\sim 20^{\circ} \mathrm{C}$ for up to 1 year. As expected, the material processed to the greatest strain of $\varepsilon_{\mathrm{eff}} \sim 10$ (15 passes ECAE) exhibited the highest hardness immediately after ECAE processing (192 HV), which was about twice that of the solution-treated control sample. For this starting condition the hardness initially increased very slightly before decreasing gradually with time. In comparison, the hardness of the undeformed, as-solution-treated sample rose rapidly from 84 to $104 \mathrm{HV}$ in two weeks and then continued to increase steadily at a slow rate as ageing continued, reaching a value of $121 \mathrm{HV}$ after 12 months. The age-hardening response of the sample processed to five ECAE passes was found, unsurprisingly, to lie between that of the as-solution-treated and the most heavily deformed conditions.

\subsection{Precipitation in the severely deformed samples}

The room-temperature hardness behaviour of the solution-treated ECAE-processed $\mathrm{Al}-4 \% \quad \mathrm{Cu}$ samples, described above, was initially thought to result from a combination of slight natural ageing, by GPZ formation, and softening due to longer-term recovery of the deformation structure. However, microstructural analysis revealed that fine scale precipitation had occurred on grain boundaries within the ECAE-processed materials after three weeks. 


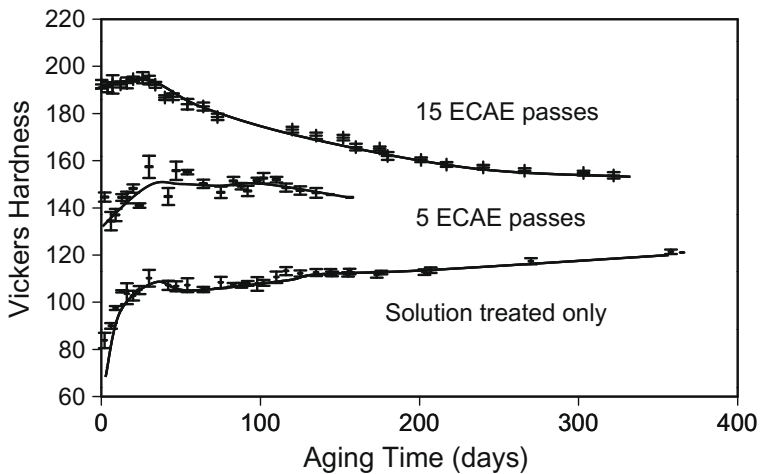

Fig. 3. Microhardness as a function of natural ageing time (at $\sim 20^{\circ} \mathrm{C}$ ) for samples, only solution-treated (undeformed), and following solution treatment and processing by ECAE to a total of five and 15 passes.

In contrast, no evidence of room-temperature grain boundary precipitation was seen in the undeformed solution-treated alloy even after natural ageing for up to a year (Fig. 4).

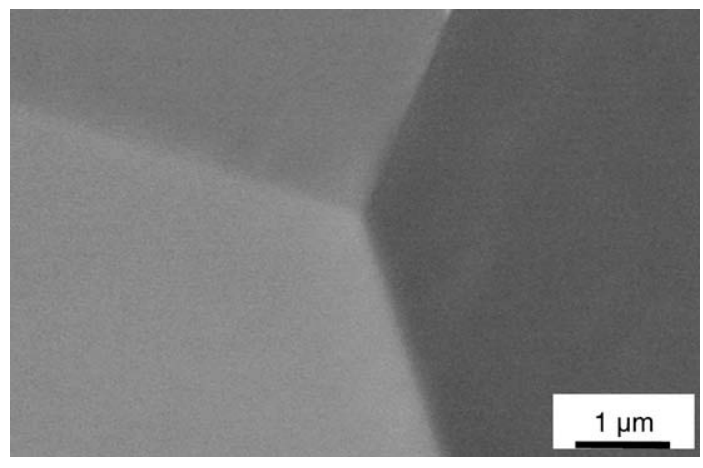

Fig. 4. High-resolution BSE images of the solution-treated undeformed control sample, showing no evidence of room-temperature grain boundary precipitation following 10 months' natural ageing.

\subsubsection{Precipitation after 15 ECAE passes}

The BSE images in Fig. 5 show the distribution of second-phase particles found in the sample processed by
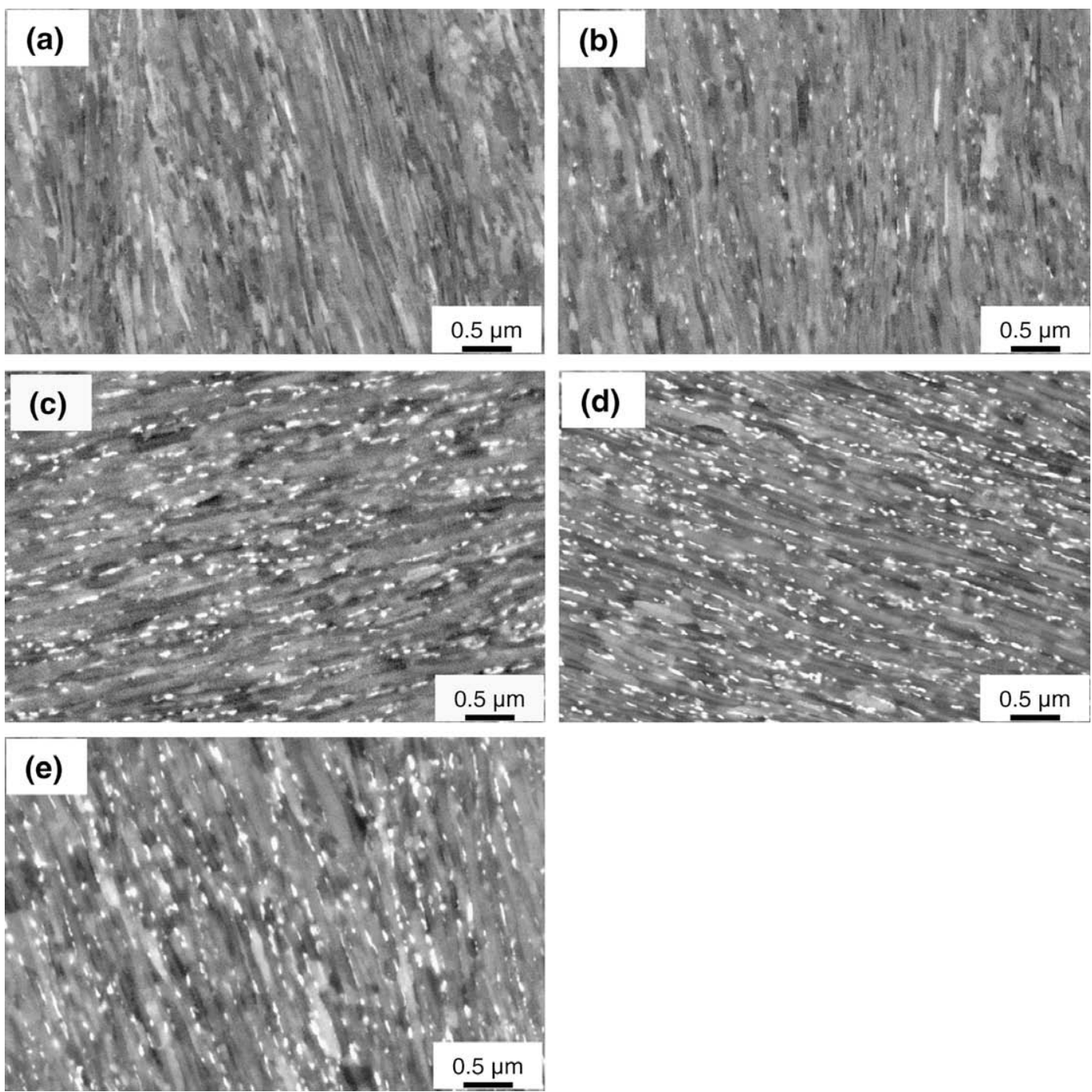

Fig. 5. Electron backscatter images of the $\mathrm{Al}-4 \mathrm{Cu}$ sample processed to $15 \mathrm{ECAE}$ passes, showing the size and distribution of second-phase particles within the deformation structure after natural ageing at room temperature for: (a) 0 days (as deformed), (b) 21 days, (c) 45 days, (d) 90 days, (e) 180 days and (f) 360 days. 

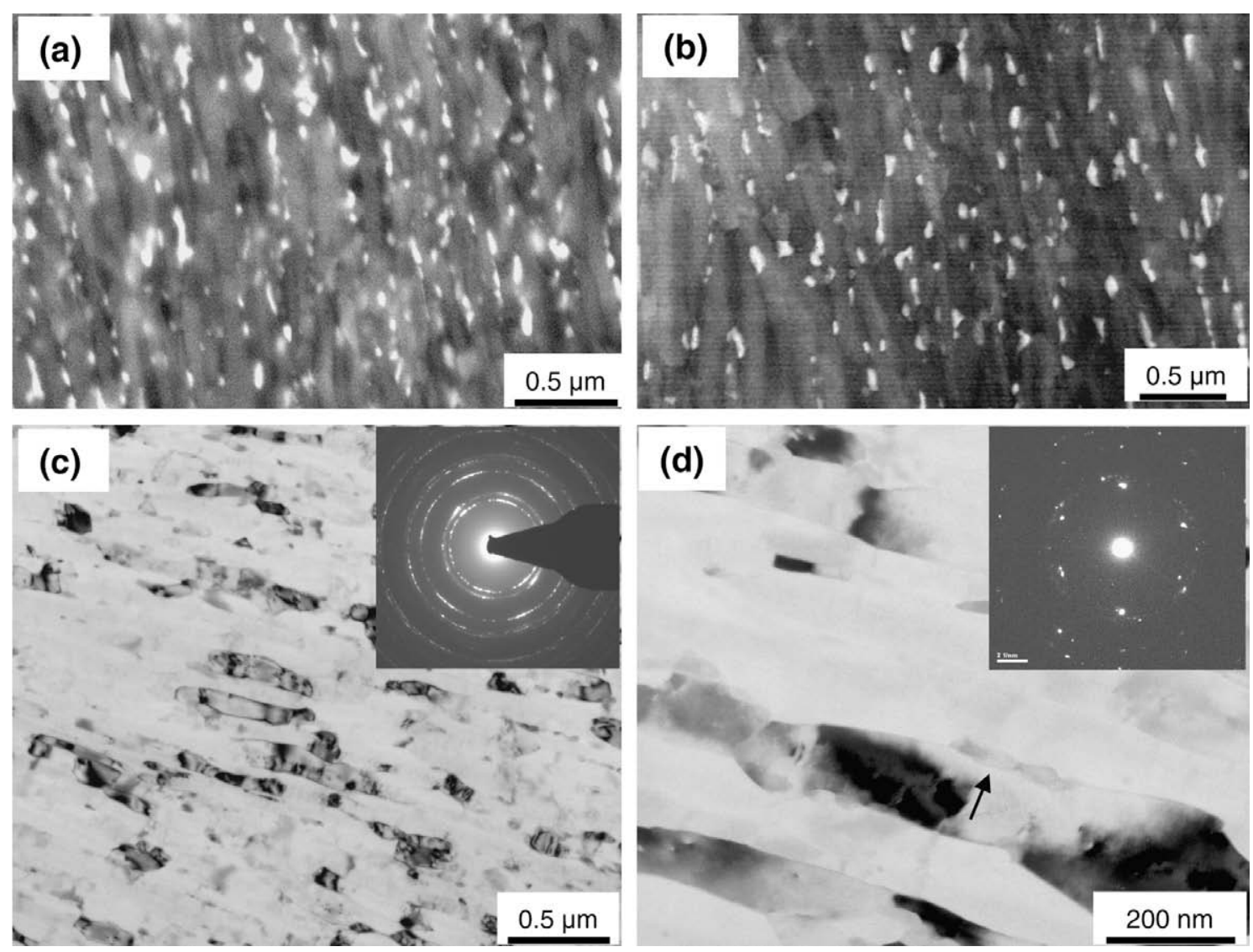

Fig. 6. High-resolution images of the recovered deformation structure and precipitate distributions in the 15 pass ECAE-processed sample after ageing at room temperature for 10 months', revealed by: (a) BSE imaging in a FEGSEM, (b) HAADF-STEM and (c and d) TEM BF images at two magnifications with corresponding selected area diffraction patterns.

ECAE to 15 passes during ageing at room temperature with increasing time. Their morphology is depicted in more detail in Fig. 6 after 10 months' ageing. Although no precipitation was observed immediately after processing (Fig. 5a), small precipitates were first observed by high-resolution FEGSEM BSE imaging at the end of the third week (Fig. 5b), when the hardness was close to its peak. All the precipitates were found to have nucleated on grain boundaries, and have a roughly elliptical shape with their long axes in line with the grain boundary plane. With increased ageing time, their number density increased and those previously formed continued to grow.

Generally, incoherent grain boundary precipitates in Al- $\mathrm{Cu}$ alloys are found to be the equilibrium $\theta$ phase $[36,37]$. To confirm this supposition, X-ray diffraction was carried out on an as-solution-treated sample and a specimen processed by 15 ECAE passes and stored at room temperature for 10 months'. Careful analysis of the diffraction data revealed only the presence of the equilibrium $\theta$ phase in the ECAE-processed sample.

The length, width, number density, and volume fraction of $\theta$ precipitates with natural ageing time were measured from BSE images and are shown in Fig. 7. It should be noted that with optimum conditions the spatial resolution of BSE images in the FEI Sirion FEGSEM under ultra- high-resolution mode is $\sim 5 \mathrm{~nm}$, which leads to a level of uncertainty in the data for times less than 30 days, where the average precipitate widths were less than $15 \mathrm{~nm}$ (length $50 \mathrm{~nm}$ ). Samples were, therefore, also examined in the TEM. However, with conventional TEM imaging it was extremely difficult to obtain statistically reliable data as it was not trivial to distinguish the second particles from the fine matrix crystallites. There were also no diffraction conditions where the $\theta$ particles in a local area could be exclusively revealed in dark field, due to the small scale and wide spread of orientations of the matrix grains. Selected samples were therefore examined by HAADF imaging using an FEI Tecnai F30 FEGTEM operated in STEM mode. Examples, comparing these imaging modes, are given in Fig. 6 for the sample stored at room temperature for 10 months' after ECAE processing by 15 passes. It can be seen that at this ageing stage both BSE and STEM images have revealed the second-phase particles clearly with adequate spatial resolution. No evidence of GP zones, or $\theta^{\prime \prime}$ and $\theta^{\prime}$ precipitates, with a coherent or semi-coherent relationship with the matrix, could be identified in the TEM images or selected area diffraction patterns where they should cause streaking in the matrix reflections and any additional diffraction spots (see the diffraction pattern inserts in Fig. 6). 
(a)

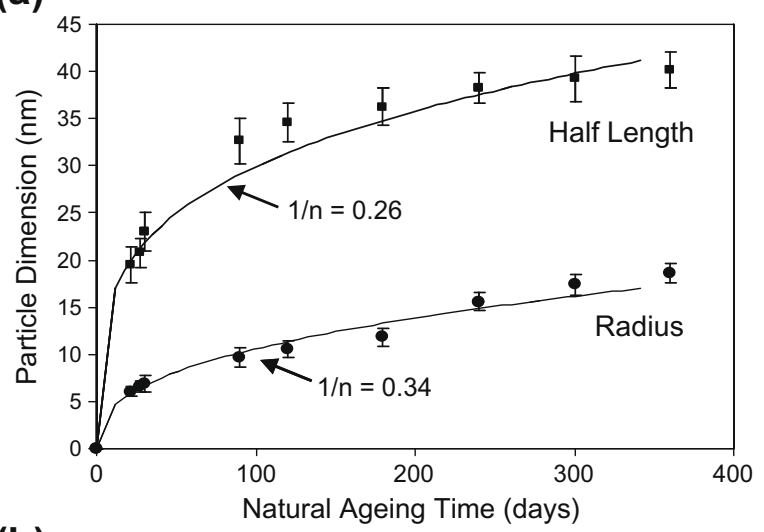

(b)
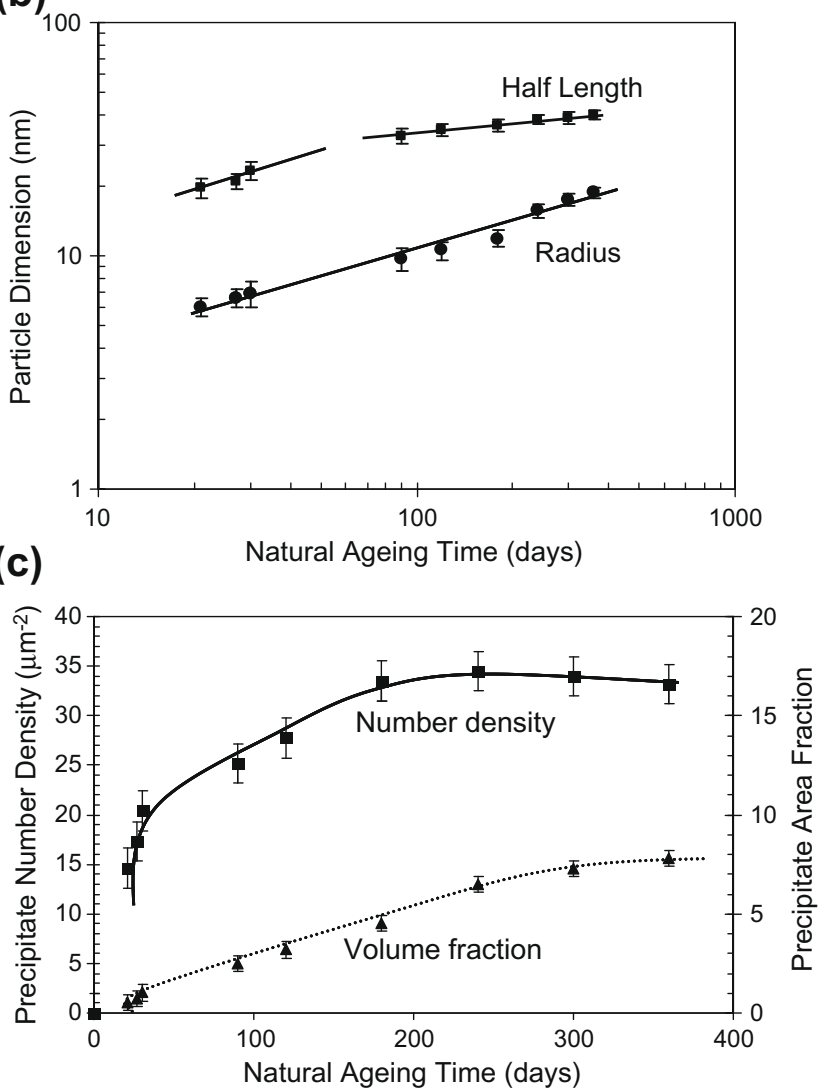

Fig. 7. Measurements of average $\theta$ precipitate dimensions (radius or half width $r$, and half length $L$ ) from the sample subjected to 15 ECAE passes, as a function of natural ageing time (a) and re-plotted logarithmically (b), showing evidence of two slopes with $1 / n \sim 0.35$ and $1 / n \sim 0.14$ for the precipitate half length results. In (c) the change in precipitate volume fraction (area fraction) and number density measured from high-resolution BSE images is also plotted against time. In (a) the continuous lines are fitted curves with $t=k_{1} r^{0.38}$ and $t=k_{2} L^{0.26}$.

\subsubsection{Kinetic data}

$\theta$ precipitate particle size data, showing the precipitates half length $(L)$ and half width $(r)$, are plotted in Fig. 7 as a function of ageing time. It can be seen from Fig. 7a that the precipitate dimensions increase at a diminishing rate with time. However, after 1 year of ageing the average precipitate width and length had increased to 37 and $80 \mathrm{~nm}$, respectively. Linear regression fits to all the data, when plotted logarithmically (Fig. 7b), gave exponents of $r^{0.38}$ and $L^{0.26}$ and curves with these exponents give good fit to the data in Fig. 7a. This is in close agreement with the $t=k_{1} L^{1 / 4}$ and $t=k_{2} r^{1 / 2}$ relationships originally proposed by Aron and Aaronson for collector plate growth of a grain boundary phase [38], although their experimental data suggested a relationship of $t=k_{2} r^{1 / 3}$, which they attributed to partial coherency at the interface. The results in Fig. 7, therefore, follow the expected behaviour for diffusion-controlled grain boundary growth. However, due to the close spacing of the precipitates within the boundary plane and simultaneous recovery of the deformation structure, the behaviour is not as straightforward as this simple fit implies. In Fig. $7 \mathrm{~b}$ it can be noted that the lengthening results show evidence of two slopes, the first implying $1 / n \sim 0.35$ and the second $1 / n \sim 0.14$.

The area fraction and number density of the $\theta$ precipitates with time are also given in Fig. 7c, where it can be seen that both parameters approach a constant level after $\sim 9$ months. This suggests that the solute supersaturation has decayed and approached the equilibrium concentration for copper in solid solution by this stage. The final area fraction of the $\theta$ phase was $\sim 7 \%$ which, given the uncertainty in the measurements, is consistent with an equilibrium volume fraction predicted from thermodynamic calculations of $\sim 5 \%$ [39].

\subsubsection{Precipitation in less severely deformed samples}

$\theta$ precipitation was also found to occur during natural ageing in the samples processed by ECAE to lower strains. Fig. 8 shows example BSE images obtained from samples after ECAE processing to two and seven passes, following 4 months' natural ageing at room temperature. Even in the sample processed to low strains (Fig. 8a and c), $\theta$ precipitates were found locally within intense shear bands, where new deformation-induced high-angle boundaries were observed. With increased strain, or number of ECAE passes, more precipitates were observed, again predominantly within shear bands, which affected a larger volume of the material, thus giving a more uniform distribution (Fig. 8b). From these observations it was apparent that the precipitation kinetics were directly related to the density and character of the boundaries generated during ECAE processing. An example of this behaviour can be seen from comparing the higher magnification image of $\theta$ boundary precipitation in Fig. $8 \mathrm{c}$ with the corresponding EBSD map from the same area in Fig. 8d where the misorientation of individual boundaries are shown.

$\theta$ particle sizes were also measured as a function of strain after 4 months' natural ageing and the results are given in Fig. 9. It can be seen that the average precipitate size rose by $\sim 70 \%$ on increasing the number of ECAE passes from 2 to 15 . This behaviour can be correlated to the increase in average misorientation of the deformation-induced HAGBs present, which doubles with deformation in this strain range from $\sim 20^{\circ}$ to $40^{\circ}$ (Table 1 and Fig. 9). 

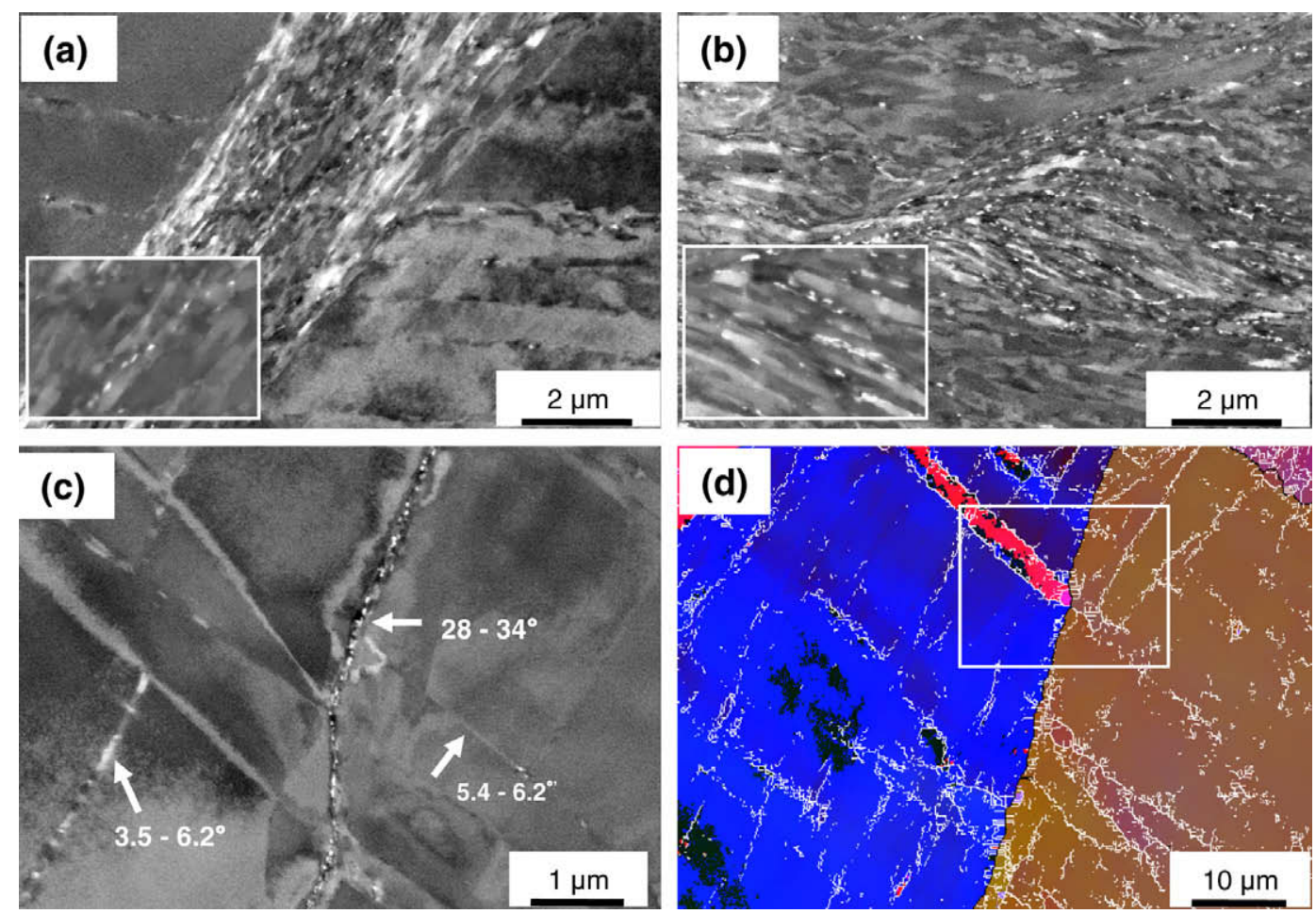

Fig. 8. Electron backscatter images obtained from samples naturally aged at room temperature for 4 months after ECAE processing to: (a) two passes, (b) seven passes, showing the dependence of the local precipitation behaviour on the heterogeneity of the deformation structure, and (c) at a higher magnification after two passes following 4 months' natural ageing, showing precipitation predominantly at higher misorientation boundaries within the deformation structure, revealed by EBSD (d).

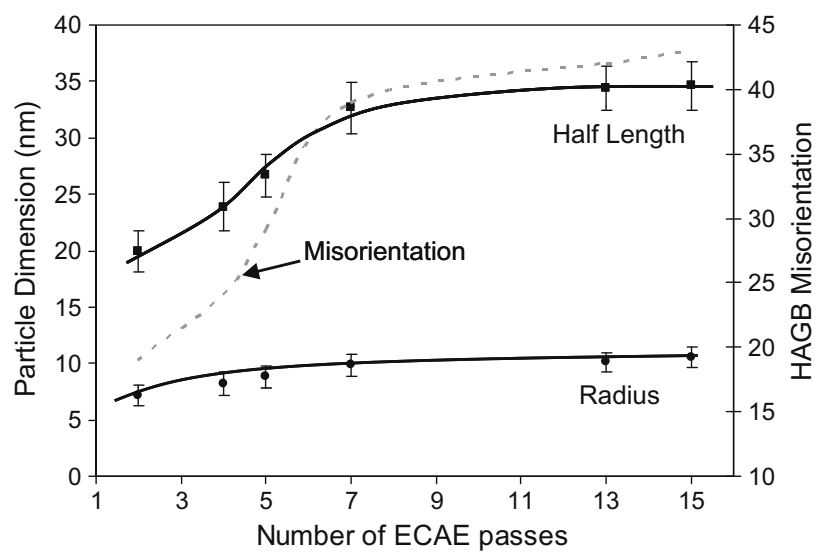

Fig. 9. Average $\theta$ precipitate size (radius or half width $r$, and half length $L$ ) and misorientation of the deformation-induced HAGBs present, as a function of strain after 4 months' ageing at room temperature.

\subsection{Recovery during natural ageing}

During prolonged natural ageing, moderate coarsening of the nanocrystalline lamellar grain structure was detected in the samples subjected to ultra-high strain deformation. The increase in lamellar boundary spacing $\left(\lambda_{\mathrm{ND}}\right)$ with ageing time after 15 ECAE passes $\left(\varepsilon_{\text {eff }} \sim 10\right)$ was measured from BSE images in the FEGSEM and the results are shown in Fig. 10, along with the increase in precipitate volume fraction. The points shown as open diamonds were

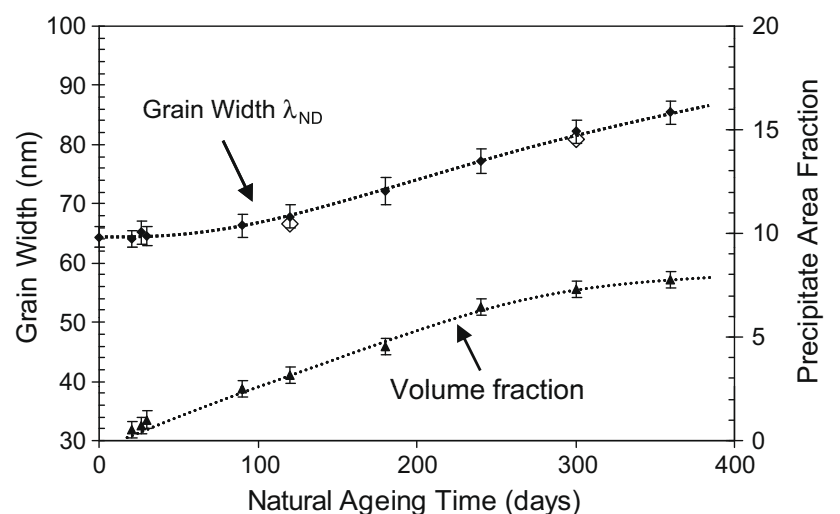

Fig. 10. Change in the lamellar boundary spacing $\left(\lambda_{N D}\right)$, or grain width, and $\theta$ phase volume fraction measured from BSE images with time at room temperature after ECAE processing by 15 extrusion cycles. The points shown as open diamonds are from TEM measurements.

obtained from TEM measurements and are very close to the values from the BSE images. From Fig. 10 it can be seen that after 1 year's natural ageing the average lamellar boundary spacing increased from $65 \mathrm{~nm}$ to $85 \mathrm{~nm}$. Slow coarsening of the lamellar structure had thus occurred, despite a simultaneous increase in the volume fraction and density of pinning particles. This coarsening of the grain structure during post-processing recovery can thus be attributed to a weakening of the solute pinning effect of copper on grain boundaries, as a result of precipitation 
and growth of the $\theta$ phase lowering the copper level in solid solution.

\section{Discussion}

\subsection{Deformation structure evolution}

The evolution of deformation structures during ECAE processing via route A (no billet rotation) has been previously extensively investigated in very dilute aluminium alloys at room temperature $[2,6,7]$, but less well studied in materials containing high levels of solute, where cellular structures are not expected to form at low strains [22]. In SPD the increase in new high-angle grain boundary (HAGB) area with strain originates from two main grain subdivision processes: deformation banding (or orientation splitting) and shear banding (which predominantly occurs at low stains <3) [6,7]. With deformation processes like ECAE by route $\mathrm{A}$, which involves a continuous material element distortion, there is also a geometrical increase in HAGB area as the grain structure elongates and compresses with strain $[3,6,7]$ to form a lamellar structure. In ECAE using route $\mathrm{A}$ this is an important factor in creating new HAGB area and the spacing of the boundaries will reduce due to this effect alone, until they converge with the subgrain size forming thin ribbon grains by a strain of $>7[2,6,7]$. At higher strains the microstructure continues to refine by the break-up of the ribbon grains into lower aspect ratio submicron grains. This occurs inhomogeneously and starts to develop locally in bands at strains as low as $\varepsilon_{\mathrm{vm}} \sim 4$ [6]. More recently it has been shown, in dilute alloys, that the transverse boundary spacing approaches a steady state at strains greater than ten, controlled by equilibrium between the rate of geometric compression of the boundaries and dynamic boundary migration $[3,6]$. However, with continued deformation there is still a slow reduction in grain length. The steady state and the progressive break-up of the ribbon grains is now thought to involve dynamic boundary migration to equilibrate surface tensions at boundary nodes and probably occurs in a manner related to geometric dynamic recrystallization (GDR) seen at higher temperatures [18].

In the work presented here with a higher solute-containing alloy it has been confirmed that copper is more effective in inhibiting recovery than magnesium, when used at a similar addition level. The average grain widths, or spacing of the lamellar boundary structures in the Al-1.7 at.\% $\mathrm{Cu}$ alloy studied, was found to be only $\sim 70 \mathrm{~nm}$ after deformation to a strain of ten. This is similar to the value of $\sim 100 \mathrm{~nm}$ reported by Murayama et al. for an alloy of similar composition after ECAE processing [29], and is less than that observed in an Al-3.3 at.\% Mg alloy deformed under identical conditions [11]. In alloys where the solute has a very strong effect on inhibiting recovery, less dynamic boundary migration occurs and the lamellar structure is thus retained to higher strains and has a finer spacing. Therefore, while it is possible to obtain much finer defor- mation structures by using solute to inhibit recovery in SPD, the resultant grain structures tend to be highly elongated and contain thinner, much higher aspect ratio grain fragments. Such ultra-high strain deformation structures only approach the nanoscale in the transverse direction, and contain lamellar, or lathe shaped, grains, with grain fragments retained within the deformation structure having lengths greater than one micron not being uncommon.

Processing high-solute-content alloys also affects the relative contributions of the different grain subdivision processes occurring during SPD. Although shear banding has previously been identified as an important mechanism of grain refinement during ECAE processing of dilute aluminium alloys (e.g. Al-0.1 wt. \% Mg) it normally only initiates in the second pass, and a more significant proportion of new HAGBs are formed by other mechanisms, such as orientation splitting [6]. In comparison in the $\mathrm{Al}-\mathrm{Cu}$ alloy studied shear banding was more intense and could be identified as the dominant mechanism in generating new HAGBs at lower strains. This is not that surprising, as it is well known that shear banding is promoted in materials where strong solute dislocation interactions occur [40]. Moreover, the presence of shearable solute clusters, or early stage GPZs, will further encourage flow instability. This behaviour is also promoted by the ECAE technique, which by the nature of the die design encourages shear banding due to the narrow intense deformation zone $[16,17]$. Furthermore, with route $\mathrm{A}$ in each subsequent extrusion cycle the principal shear plane alternates through a rotation of $60^{\circ}$ about TD (with a $120^{\circ}$ die) [16] and this reorientation of the shear plane also promotes shear banding within the billet [41].

\subsection{Accelerated natural ageing}

Deformation-induced dynamic precipitation during SPD processing has been previously reported in certain aluminium alloys [32]. It is also well known that prior deformation can greatly accelerate static artificial ageing (e.g. Refs. [34,42]). Under the extreme strain levels reached in ECAE and other SPD techniques, dynamic precipitation, or post-deformation static ageing, are therefore not unexpected, particularly as plastic work can raise the temperature during processing to around $50{ }^{\circ} \mathrm{C}$. However, the precipitation processes that take place will depend on the defect structure created, including the new high- and lowangle boundary area and statistically stored dislocations, the chemical driving force, or supersaturation, and the strain rate, temperature and activation energies for the dominant diffusion processes involved. A further contributory factor is the possibility of enhanced diffusion owing to the presence of an artificially high, deformation-induced, vacancy content. This is an important consideration and vacancy concentrations have been estimated to be as large as $10^{-4}$ immediately after severe deformation processing [33] compared to an equilibrium concentration of $\sim 10^{-10}$ [43]. In a material containing a high density of vacancy 
sinks, resulting from a fine grain size, this excess vacancy concentration might be expected to decay rapidly. However, in a coarse-grained $\mathrm{Al}-\mathrm{Cu}$ alloy, positron annihilation lifetime spectroscopy (PALS) has shown that, although the vacancy concentration after quenching decays to half of its original value within a few hours, it then continues to decrease relatively slowly, approaching equilibrium over several weeks [44].

Having carefully examined the microstructure of the ECAE-processed billets immediately after deformation (Fig. 1), it was found that precipitation only occurred some time after processing. In comparison, no grain boundary precipitation was detected even after a year in an identically room-temperature-aged undeformed control sample (Fig. 4). Therefore, the observations reported are solely related to the acceleration of static precipitation by prior severe deformation. Precipitation in $\mathrm{Al}-\mathrm{Cu}$ alloys, and of the $\theta$ phase at specific sites such as grain boundaries, has been previously extensively investigated [37,38,45-47], although all of this work was carried out during artificial ageing at higher temperatures, typically greater than $170{ }^{\circ} \mathrm{C}$. The only exceptions to this research are the results of Murayama et al. [29] who, on ageing an equivalent alloy at $100{ }^{\circ} \mathrm{C}$, also noted $\theta$ precipitation at grain boundaries after ECAE processing to a similar strain level. Murayama et al. further showed that the metastable transition phases normally seen on ageing $\mathrm{Al}-\mathrm{Cu}$ alloys did not form in the SPD-processed material, due to the high nucleation site density for $\theta$ precipitation. Although we have focused on the theta phase, no indication of $\theta^{\prime}, \theta^{\prime \prime}$ or GPZ formation was observed in any of the severely deformed samples examined in our experiments. Therefore, a similar behaviour to that reported by Murayama et al. [29] was also seen in the current work, but over a longer time scale at room temperature, with the solid solution decaying directly by the formation of $\theta$ bypassing the normal transition phase states.

After $\sim 9$ months' natural ageing full decomposition of the solid solution was observed in the severely deformed samples which, given the average grain width, would require a diffusion distance of $\sim 40 \mathrm{~nm}$. An indication of the upper bound on the level of acceleration of the kinetics required to achieve this loss of solute from the matrix can be obtained by estimating the time for copper to achieve this diffusion distance by normal lattice diffusion at room temperature $\left(20^{\circ} \mathrm{C}\right)$, which is about 850000 years. This calculation was based on the root mean diffusion distance $(\bar{x}=\sqrt{D t})$ using the recommended diffusion data assessed by $\mathrm{Du}$ et al. [32] $\left(D=4.44 \times 10^{-5} \exp .(-133,900 / R T)\right)$. The available diffusion data highlights another problem, in that there is a wide range of values in the literature, none of which was determined below $250^{\circ} \mathrm{C}$.

It is further interesting that nucleation of $\theta$ also occurs after processing to relatively low bulk strains at specific sites within the deformation structure (Fig. 8a). This is a direct consequence of the localization of shear promoted by the ECAE process which results in intense strain con- centration within shear bands. In such regions precipitation is also accelerated because the local environment, in terms of the presence of new deformation-induced HAGBs for nucleation and the vacancy content, is already similar to that seen in the whole sample after ultra-high strain deformation.

Consideration will now be given to the $\theta$ phase grain boundary nucleation and growth stages, with predictions made from classical nucleation and growth theories used as a framework for discussing the observed accelerated ageing kinetics.

\subsubsection{Grain boundary nucleation of $\theta$ phase at room temperature}

Heterogeneous nucleation on grain boundaries is classically described in terms of the reduction in energy barrier that results from destruction of grain boundary area, when the boundary plane is wetted by the nucleus [48]. Incoherent phases like $\theta$, which have a poor crystallographic fit to the matrix and a high interfacial energy, benefit most from nucleating at grain boundaries, whereas semi-coherent phases cannot nucleate on grain boundaries and maintain their habit plane relationship unless the boundary misorientation is small $\left(<9^{\circ}\right.$ in the case of $\theta^{\prime}$ [37]). Although the average energy of a boundary generally increases with misorientation, and this should encourage nucleation, more important is the energy and density of specific sites within the grain boundary plane. In a fully relaxed boundary this is often related to the CSL (coincident site lattice), or O-Lattice [47] models, and it has been argued that for non-special boundaries the site potency will first increase with misorientation and then decrease again at higher misorientations as the site density becomes too high and the energy per site becomes diluted. However, fully relaxed boundaries would be expected to provide less effective nucleation sites than the boundaries present after deformation, which will contain steps, and generally be in a more disordered state $[1,49]$. The presence of "non-equilibrium" deformation-induced boundaries has also been previously used to try to explain other phenomena in severe deformation-processed materials, such as more rapid grain coarsening due to enhanced grain boundary diffusion [13].

Although there are many problems with classical nucleation theory, not least of which is the high sensitivity to the interfacial energy, here we have used this approach to make ball-park estimates of grain boundary nucleation times for the $\theta$ phase, in order to identify the factors that control the accelerated nucleation rate seen following SPD. Conveniently, the theory of diffusion-controlled grain boundary nucleation developed by Russell $[38,45]$ was originally applied to predict the incubation times for grain boundary nucleation of the $\theta$ phase. In this work it was shown that the data were consistent with a nucleation mechanism controlled by volume diffusion of solute into and out of the nucleus, rather than grain boundary diffusion. Following Russell, for a cap-shaped nucleus geometry, the incubation time for nucleation $(\tau)$ is given by: 


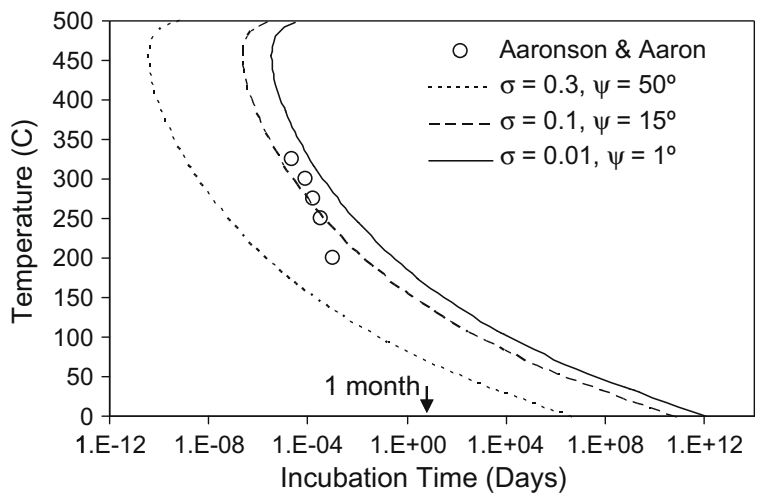

Fig. 11. Predicted incubation times for nucleation of grain boundary $\theta$ precipitates as a function of temperature, from classical nucleation theory. Three assumptions about the nucleus interfacial energy $(\sigma)$ and contact angle $(\psi)$ were used to perform the calculations (see text). Also shown are the measured incubation times of Aaron and Aaronson, as reported by Russell [45].

$\tau=\frac{k T \sigma_{\alpha \theta} a^{4}}{V_{\alpha}^{2} \Delta G_{v}^{2} D_{v} \bar{c}} \frac{\left(2-3 \cos \psi+\cos ^{3} \psi\right)}{(1-\cos \psi)}$

where $k$ is Boltzmann's constant, $T$ is absolute temperature, $\sigma_{\alpha \theta}$ is the interfacial energy of the nucleus/matrix interface, $a$ is the matrix lattice parameter, $V_{\alpha}$ is the atomic volume of the copper solute, $\Delta G_{v}$ is the volume free energy change for formation of $\theta, D_{v}$ the diffusion coefficient of copper in the aluminium matrix, $\bar{c}$ the mole fraction of solute in the matrix, and $\psi$ the nucleus contact angle.

It is well known that this theory is extremely sensitive to the shape of the nucleus and the contact angle $\psi$, which is determined by the requirement for balance between the surface tensions of the nucleus and grain boundary. In Fig. 11, Eq. (1) has been applied to estimate the expected incubation time at room temperature $\left(20^{\circ} \mathrm{C}\right)$ using various assumptions about the nucleus shape and interfacial energy. Also plotted on this figure are the measured incubation times of Aaron and Aaronson [38], as reported by Russell [45].

The first assumption we have used is the same as that adopted by Brailsford and Aaron [50], where it was assumed that the contact angle $(\psi)$ and interfacial energy $\left(\sigma_{\alpha \theta}\right)$ for the nucleus are the same as those for growing $\theta$ precipitates $\left(\psi=50^{\circ}, \sigma_{\alpha \theta}=0.3 \mathrm{~J} \mathrm{~m}^{-2}\right)$. Using these values gives the rightmost curve in Fig. 11, corresponding to the longest incubation times. For room-temperature ageing, this gives an estimated incubation time for grain boundary nucleation of $7.5 \times 10^{7}$ years! It can be seen that using this assumption also overestimates the incubation time relative to Aaron and Aaronson's data at elevated temperatures.

While appropriate for growth, it could be argued that the value of interfacial energy of $0.3 \mathrm{~J} \mathrm{~m}^{-2}$ is too large for nucleation, since the interfacial structure of small nuclei will be significantly different to that of larger precipitates with a well-defined crystal structure [51]. A reduction in the nucleus/matrix interfacial energy would necessarily lead to a change in the nucleus shape to maintain equilib- rium at the contact with the grain boundary. Reducing the interfacial energy to $0.2 \mathrm{~J} \mathrm{~m}^{-2}$ would require $\psi=15^{\circ}$ to maintain equilibrium. This represents the case where wetting of the grain boundary by the nucleus is greatly enhanced relative to that of a growing precipitate, thus reducing the incubation time. Using these parameters produced the middle curve in Fig. 11, and gives a more reasonable fit to the Aaron and Aaronson measured incubation times at $250{ }^{\circ} \mathrm{C}$ and above. However, when the curve is extended to room-temperature ageing, the predicted incubation times still remain far too long $\left(\sim 1.4 \times 10^{6}\right.$ years $)$ to explain the measured nucleation kinetics. If the requirement for balance between surface tensions at the contact point is ignored, and very low values are used for the interfacial energy and wetting angle (e.g. $\sigma_{\alpha \theta}=0.01 \mathrm{~J} \mathrm{~m}^{-2}$, $\psi \sim 1^{\circ}$ ), a much shorter incubation time is predicted (the left-hand curve in Fig. 11). Nevertheless, even using this extreme set of conditions, which are unrealistically favourable for nucleation, the incubation time at room temperature is still predicted to be in excess of 80 years, whereas in practice small precipitates were observed after less than a month in the severely deformed material.

The above analysis demonstrates that at low temperatures, the incubation time is largely controlled by atomic mobility and the prediction of very long incubation times at room temperature is dominated by the low diffusion coefficient term in Eq. (1). At low temperatures the availability of potent grain boundary nucleation sites will thus affect the spatial distribution of nuclei, but not control the nucleation rate. The calculations used to produce Fig. 11 were based on the most reliable data available from the recent assessment of the diffusivity of copper in aluminium, by Du et al. [32]. However, even if the predictions are made using an upper bound values for $D$, the incubation time at room temperature remains several years, rather than the month observed. This indicates that other factors are important in controlling nucleation in the severely deformed samples that are not accounted for in the Russell model for grain boundary nucleation.

Additional factors that could accelerate nucleation are a higher copper content in the grain boundaries, as forced migration of boundaries during deformation may result in stronger copper segregation from the swept matrix, which would increase the driving force and local availability of solute. Vacancy flux effects [52] are known to have an important accelerating influence on the nucleation of precipitates in quenched aluminium-magnesium-zinc alloys [53]. However, it is clear that the present observations cannot be explained by this thermodynamically induced segregation alone, since this does not explain the large difference observed between the kinetics in the heavily deformed and undeformed samples. More important, given the dominance of mobility over the driving force, is the possibility of diffusion being enhanced by contributions from the high initial deformation-induced vacancy concentration and the more disordered nature of the boundaries and associated extrinsic dislocation structures. A temporary $10^{6}$ rise in 
vacancy content is consistent with that expected immediately after severe deformation [33], and could produce an increase in mobility sufficient to explain how room-temperature nucleation is possible, even when using the more realistic second set of assumptions detailed above. Although the vacancy concentration will rapidly decay, this is probably still the most significant factor encouraging nucleation in the SPD-processed material, as predictions of the nucleation rate retain large levels of uncertainty and PALS measurements show that there will be an enhanced vacancy content, albeit at a reducing level, over a similar period to that required for the first precipitates to become visible [44].

\subsubsection{Room-temperature growth kinetics}

To prove that accelerated growth, as well as nucleation, of the theta phase was occurring in the SPD-processed materials, and identify the factors that might be involved, we have similarly compared predictions from the classical collector plate theory of the growth of a grain boundary nucleated phase to our measured data.

A number of models exists describing the growth rate of grain boundary nucleated phases that follow on from the original pioneering work by Aaron and Aaronson [38]. Subsequent modifications have been proposed by Brailsford and Aaron [50], Cantrell and Shiflet [46] and Faulkner and co-workers [53,54]. The original data used by Aaron and Aaronson were for the same alloy composition investigated here, but is now quite old and relied on optical microscopy performed on samples heat-treated above $200{ }^{\circ} \mathrm{C}$. However, more recent results, on the effect of boundary misorientation [46] and in thin Al-Cu films [55], still show good agreement with this model. Encouragingly, our data also gave good agreement with the exponents predicted by this theory for the lengthening rate $(1 / n \sim 0.25)$, and thickening $(1 / n \sim 0.5)$, which is largely controlled by interfacial diffusion at low temperatures. Brailsford and Aaron [50] have further demonstrated that the exponents can lie in the range $0.25-0.37$ for lengthening, and $0.26-0.34$ for thickening, depending on the temperature.

Here we have used a version of the theory with a constant collector plate area, as proposed by Carolan and Faulkner [54], to estimate the expected growth rates of the $\theta$ phase at room temperature, which is given by:

$\frac{d L}{d t}=\frac{A_{v} \sqrt{D_{v}}\left(\bar{x}-x_{r}^{\alpha \theta}\right)}{3 \pi^{3 / 2} f(\psi)\left(x^{\theta}-x_{r}^{\alpha \theta}\right)} \frac{\sqrt{t}}{L^{2}}$

where $A_{v}$ is the collector plate area, $\bar{x}$ the volume fraction of copper in the matrix, $x_{r}^{\alpha \theta}$ the volume fraction of copper at the precipitate/matrix interface corrected for curvature (the Gibbs-Thompson effect), $x^{\theta}$ the volume fraction of copper in the precipitate, $f(\psi)$ a geometric factor (the expression for which is given in Ref. [54]), $L$ the precipitate half length and $t$ time.

This model has been shown to give predictions that are at least as good as the Aaron and Aaronson model, with the benefit of greater mathematical simplicity. As in the
Table 2

Input parameters to the Russell model for grain boundary precipitate growth. The original values are those used by Brailsford and Aaron [50]. The modified values are for a more realistic estimate of the collector plate area in the SPD-processed material and a more recent assessment of the activation energy and pre-exponential factor for diffusion of copper in aluminium [32].

\begin{tabular}{lll}
\hline Parameter & Original value & Modified value \\
\hline Collector plate area, $A_{v}$ & $12.57 \mathrm{~m}^{2}$ & $0.05 \mu \mathrm{m}^{2}$ \\
Diffusion activation energy, $Q$ & $129.7 \mathrm{~kJ} \mathrm{~mol}^{-1}$ & $133.9 \mathrm{~kJ} \mathrm{~mol}^{-1}$ \\
Diffusion pre-factor, $D_{0}$ & $0.29 \times 10^{-4} \mathrm{~m}^{2} \mathrm{~s}^{-1}$ & $4.44 \times 10^{-5} \mathrm{~m}^{2} \mathrm{~s}^{-1}$ \\
Contact angle, $\psi$ & $50^{\circ}$ & Unchanged \\
Geometric factor, $f(\psi)$ & 0.25 & Unchanged \\
\hline
\end{tabular}

Aaron and Aaronson model, it is assumed that the ratecontrolling step is diffusion of solute in the matrix towards the collector plate, and that diffusion along the grain boundary is effectively infinitely rapid. Therefore, this is expected to be an upper bound prediction of the growth rate. In this work, Eq. (2) was integrated numerically to find the relationship between $L$ and $t$.

Using identical input parameters to those adopted by Brailsford and Aaron [50] (shown in Table 2) and assuming the same cap-shaped precipitate morphology, in Fig. 12a Eq. (2) is shown to reasonably reproduce the experimental data of Aaron and Aaronson, notwithstanding the large scatter in the measurements. Perhaps more surprising is that, if this prediction is extrapolated to $20^{\circ} \mathrm{C}$ without any modification to the input parameters, it fits our results for the severely deformed material remarkably well, particularly at longer times (Fig. 12b). However, this agreement is serendipitous, rather than a correct indication of the true growth kinetics, since the input parameters are unrealistic for the severely deformed alloy. In particular, the collector plate area is determined by the precipitate spacing on the grain boundary, and this is much smaller in our work than in that of Aaron and Aaronson [38]. In addition, the values used for the diffusion parameters (activation energy and pre-exponential factor) used by Brailsford and Aaron are different from those of more recent and more reliable assessments [32]. This makes a significant difference to the calculated diffusion coefficient when extrapolating to room temperature.

A more realistic assessment of the collector plate size based on the actual measured interparticle spacing (see Table 2) gives greatly reduced predicted growth kinetics, as shown in Fig. 12b (solid line) and this has a stronger effect on the growth kinetics than the relatively small change introduced by using values from more recent assessment of the diffusion coefficient (compare dotted and solid lines). With this more appropriate set of input parameters the predicted precipitate size, according to Eq. (2), is now about eight times smaller than that measured. Thus, when appropriate input parameters are used, conventional growth theory under-predicts the observed rate by around an order of magnitude, which suggests that room-temperature growth of the $\theta$ phase must also be enhanced by prior 
(a)
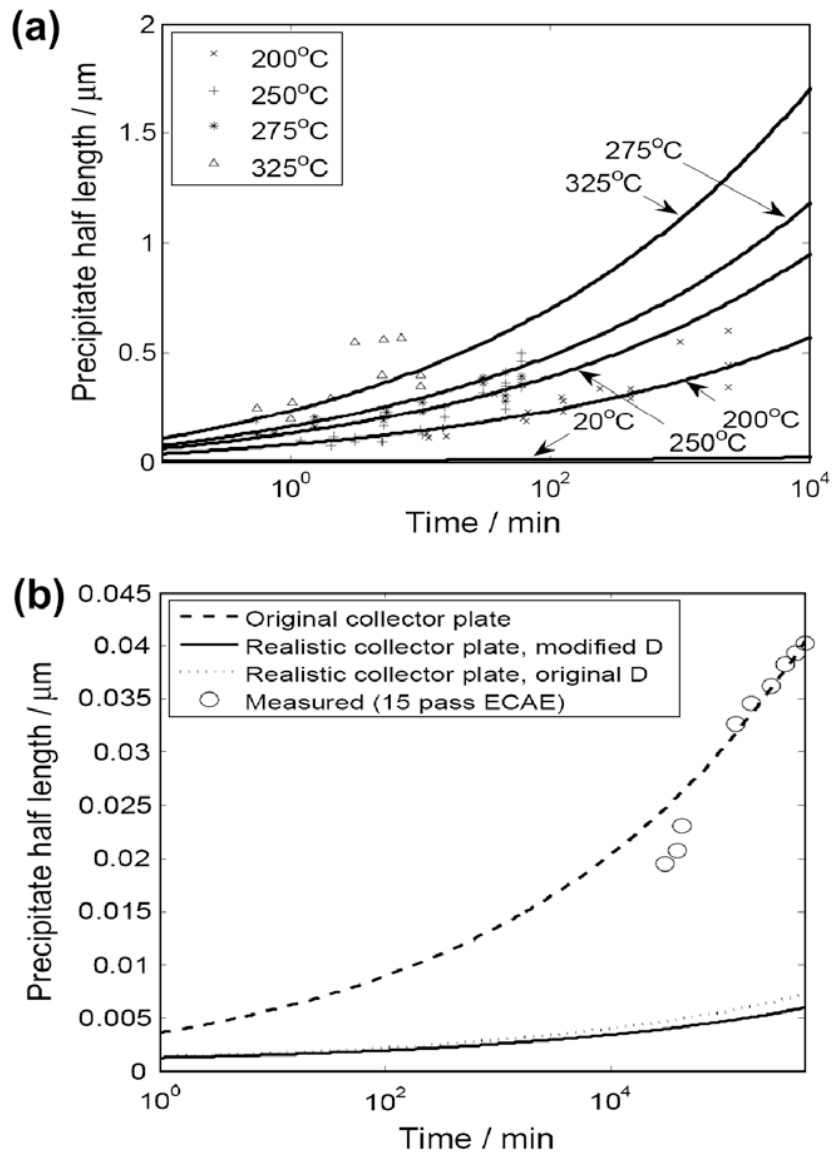

Fig. 12. The half length of grain boundary nucleated $\theta$ precipitates predicted by collector plate theory as a function of time: (a) at elevated temperatures compared to the experimental results of Aaron and Aaronson [38], and (b) at room temperature $\left(20^{\circ}\right)$ compared to data obtained in this work for material subject to 15 ECAE passes, using three different assumptions explained in the text.

severe deformation. However, this discrepancy is not as large as the factor of $\sim 10^{6}$ estimated for the nucleation kinetics, which clearly explains why $\theta$ would not normally be seen on natural ageing an undeformed alloy even if there was no growth limitation.

While not as dramatic as the influence of SPD on roomtemperature nucleation rates, this again implies that factors not accounted for in classical theory must be important in accelerating growth kinetics of grain boundary nucleated phases in heavily deformed materials. Although it can be argued that the deformation-induced excess vacancy concentration is important in providing sufficient atomic mobility to contribute to nucleation, the decay rate of even a large non-equilibrium vacancy concentration will be too short [44] to have much impact on growth occurring over a 1 year time scale. This is particularly true in a nanograined material with a high density of vacancy sinks. Cantrell and Shiflet [46] have shown a strong dependence of precipitate density and growth kinetics on grain boundary misorientation, with around a fivefold increase in precipitate size in the misorientation range $20-40^{\circ}$. They attributed this observation to the variation in nucleation site density, and energy, and bound- ary diffusivity $\left(\delta D_{b}\right)$ with misorientation. They note a peak in growth rate occurs when the boundary misorientation approaches $40^{\circ}$. We have also observed the $\theta$ precipitate size to increase with strain, which increases the average boundary misorientation after deformation (Fig. 9). However, this effect is relatively limited and would not account for the estimated order of magnitude increase in growth rate over and above that for an alloy that has random boundary misorientations.

In the collector plate theory [54] it is assumed that grain boundary diffusion is infinitely rapid relative to diffusion to the boundary and the dominant factors are the movement of solute to the boundary and the size of the collector plate. The size of the collector plate is relatively small in a severely deformed alloy, and the rate-determining step therefore is the supply of solute to the boundaries. It is possible this could be enhanced by two factors. Firstly, dislocations attached to boundaries that extend into the matrix could assist in supplying solute, although this effect would diminish as recovery occurs. Secondly, as migration of the lamellar boundaries occurred simultaneously with $\theta$ precipitation (Fig. 10), migration of segments of HAGBs, between pinning points from second-phase particles, could sweep copper atoms to boundaries, enhancing the collector plate effect. The possibility of a greater supply of solute caused by slowly migrating grain boundaries is not accounted for in the simple classical growth model and, although the potential influence of this effect requires further investigation, it is likely to be a key factor contributing towards the acceleration of the room-temperature $\theta$ growth kinetics in the SPD-processed material.

\section{Conclusions}

The grain size achievable after SPD processing and long-term stability of an aluminium copper alloy have been investigated, where copper was intentionally used in solid solution to inhibit recovery. After deformation to a strain of $\varepsilon_{\mathrm{eff}} \sim 10$, by ECAE through "route A", the deformed state contained highly elongated lamellar grain fragments with a grain width of only $70 \mathrm{~nm}$ and an average length of $\sim 1 \mu \mathrm{m}$. This compares to a grain width of $150 \mathrm{~nm}$ for an identically processed $3 \mathrm{wt} . \% \mathrm{Mg}$ alloy, which contained double the atomic concentration of solute. As well as having a greater effect than magnesium on inhibiting dynamic recovery, copper in solid solution was found to stimulate intense shear banding which became the dominant mechanism for forming new HAGBs during the early stages of ECAE processing.

Post-ECAE processing, the severely deformed Al-Cu solid solution was found to be unstable at room temperature and copious precipitation of $\theta$ occurred at grain boundaries on natural ageing, leading to recovery of the deformation structure and a loss of strength. Precipitation of GPZs, or the other transition phases, was not observed and the solute level fell to equilibrium within $\sim 9$ months. In comparison, no grain boundary precipitation at all was seen in an undeformed identically treated control material. 
The unusually rapid precipitation kinetics observed in the SPD-processed samples were compared to estimates from classical theories of grain boundary nucleation and a simplified collector plate model for growth. Analysis showed that at high underoolings the nucleation and growth rates are dominated by low atomic mobility. Nucleation of $\theta$ was thus only possible in the severely deformed alloy, due to enhanced diffusion accelerated by the high initial vacancy flux. In comparison, the excess vacancy concentration would not be expected to survive for sufficient time to have a great impact on the growth kinetics. However, estimates of the growth rate of the nanoscale $\theta$ precipitates, using a collector plate analysis, predicted an order of magnitude slower rate than that observed. It is suggested that room-temperature growth of the $\theta$ phase in the SPD-processed alloy was probably assisted by the migration of HAGBs during recovery, which would help to sweep copper atoms to the boundaries accelerating growth.

\section{Acknowledgments}

The authors would like to acknowledge the financial support of the University of Manchester EPSRC Light Alloys Portfolio Partnership (EP/D029201/1) for this project.

\section{References}

[1] Valiev ZR, Islamgaliev RK, Alexandrov IV. Prog Mater Sci 2000;45:103.

[2] Bowen JR, Prangnell PB, Humphreys FJ. Mater Sci Technol 2000;16:1246.

[3] Huang Y, Prangnell PB. Acta Mater 2008;56:1619.

[4] Hebesberger T, Stüwe HP, Vorhauer A, Wetscher F, Pippan R. Acta Mater 2005;53:393.

[5] Pippan R, Vorhauer A. Metall Mater Trans 2008;39:417.

[6] Prangnell PB, Huang Y, Berta M, Apps PJ. Mater Sci Forum 2007;550:159.

[7] Prangnell PB, Bowen JR, Apps PJ. Mater Sci Eng 2004;A375-377:178.

[8] Apps PJ, Berta M, Prangnell PB. Acta Mater 2005;53:499.

[9] Kawasaki M, Horita Z, Langdon TG. Mater Sci Eng 2009;524:143.

[10] Wang YM, Ma E. Acta Mater 2004;52:1699.

[11] Prangnell PB, Hayes JS, Bowen JR, Apps PJ, Bate PS. Acta Mater 2004;52:3193.

[12] Zahid GH, Huang Y, Prangnell PB. Acta Mater 2009;57:3509.

[13] Wang J, Furukawa M, Horita Z, Nemoto M, Valiev RZ, Langdon TG. Mater Sci Eng A 1996;216:41.

[14] Mishin OV, Jul Jensen D, Hansen N. In: Hirsch J, Skrotzki B, Gottstein G, editors. Aluminium alloys ICAA1. Germany: Wiley$\mathrm{VCH} ; 2008$. p. 114.

[15] Morris DG, Muñoz-Morris MA. Acta Mater 2002;50:4047.

[16] Segal VM. Mater Sci Eng A 1999;271:322.

[17] Bowen JR, Gholinia A, Roberts SM, Prangnell PB. Mater Sci Eng 2000;A287:87.
[18] Jazaeri H, Humphreys FJ, Bate SP. Mater Sci Forum 2006;519521:153.

[19] Prangnell PB, Berta M, Apps PJ, Bate PS. In: Bacroix B et al., editors. 2nd International conference on recrystallisation and grain growth. France: Annecy; 2004. p. 1261.

[20] Zhao YH, Zhu YT, Liao XZ, Horita Z, Langdon TG. Mater Sci Eng 2007;A463:22.

[21] Iwahashi Y, Horita Z, Nemoto M, Langdon TG. Metall Mater Trans 1998;29A:2503.

[22] Hughes DA. Acta Metall Mater 1993;41:1421.

[23] Humphreys FJ, Hatherly M. Recrystallization and related annealing phenomena. second ed. Elsevier; 1995.

[24] Zolotorevsky NYu, Solonin AN, Churyumov AYu, Zolotorevsky VS. Mater Sci Eng 2009;A502:111.

[25] Nes E, Marthinsen K. Mater Sci Eng 2002;A322:76.

[26] Schulthess TC, Turchia PEA, Gonisa A, Nieh T-G. Acta Mater 1998;46:2215

[27] Gordon P, Vandermeer RA. In recrystallization grain growth and textures. Metals Park (OH): ASM; 1966. p. 205.

[28] Frois C, Dimitrov O. Ann Chim Paris 1966;1:113.

[29] Murayama M, Horita Z, Hono K. Acta Mater 2001;49:21.

[30] Straumal B, Baretzky B, Mazilkin AA, Phillipp F, Kogtenkova OA, Volkov MN, et al. Acta Mater 2004;52:4469.

[31] Straumal B, Valiev RZ, Kogtenkova O, Zieba P, Czeppe T, Bielanska E, et al. Acta Mater 2008;56:6123.

[32] Du Y, Chang YA, Huang B, Gong W, Jin Z, Xu H, et al. Mater Sci Eng 2003;A363:140.

[33] Lechner W, Puff W, Mingler B, Zehetbauer MJ, Würschu R. Scripta Mater 2009;61:383.

[34] Zhao YH, Liao XZ, Jin Z, Valiev RZ, Zhu YT. Acta Mater 2004; 52:4589.

[35] Gill SC, Prangnell PB. Mater Sci Eng 1994;A179-A190:327.

[36] Lorimer GW. In: Russel KC, Aronson HI, editors. Precipitation processes in solids. TMS; 1976. p. 87.

[37] Vaughan D. Acta Metall 1968;16:563.

[38] Aaron HB, Aaronson HA. Acta Metall 1968;16:789.

[39] Saunders N. J JILM 2001;51:141.

[40] Duckham A, Knutsen RD, Engler O. Acta Mater 2001;49:2739.

[41] Rauch EF. Solid State Phenom 1992;23-24:317.

[42] Shih HC, Ho NJ, Huang JC. Metall Mater Trans 1996;27A:2479.

[43] Altenpohl D. Aluminium 1961;37:401.

[44] Hutchinson CR, Gable BM, Ciccosillo N, Loo PT, Bastow TJ, Hill AJ. In: Hirsch J, Skrotzki B, Gottstein G, editors. Aluminium alloys ICAA 11. Wiley; 2008. p. 788.

[45] Russell KC. Acta Metall 1969;7:1123.

[46] Cantrell MA, Shiflet GJ. Mater Res Soc Proc 1994;319:351.

[47] Cantrell MA, Shiflet GJ. Mat Res Soc Proc 1994;319:35x.

[48] Christian JW. Theory of phase transformations in metals and alloys, Part 1. Pergamon; 1975.

[49] Huang JY, Liao XZ, Zhu YT, Zhou F, Lavernia EJ. Philos Mag 2003;83:1407.

[50] Brailsford AD, Aaron HB. J Appl Phys 1969;40:1702.

[51] Robson JD, Prangnell PB. Acta Mater 2001;49:599.

[52] Anthony TR. Acta Metall 1970;37:307.

[53] Jiang H, Faulkner RG. Acta Mater 1996;44:1857.

[54] Carolan RA, Faulkner RG. Acta Metall 1988;36:257.

[55] Park M-W, Lee J-S, Lim D-G, Choi Y-G, Kwak D-J, Sung Y-M. Vacuum 2008;83:107. 